\title{
Low-cost modular PV-battery microgrid emulator for testing of energy management algorithms
}

\author{
Leong Kit Gan, Baljit Riar, Member, IEEE, Jaehwa Lee, David Howey, Senior Member, IEEE
}

\begin{abstract}
Along with the development of microgrid, the need for smart energy management systems (EMS) has become increasingly important for users to trade power effectively with the energy market. Many researchers have been developing algorithms to forecast the availability of renewable energy, load demand whilst optimising the energy flow within the given constraints. Recently, the control system for peer-to-peer (P2P) like microgrid architecture which involves complex information and communication technology has also been given much attention. However, not all of these algorithms had the privilege to be implemented and tested with real hardware, which may be attributed to the high cost involved and the safety concern on a larger system. On the contrary, this paper describes the design, build and demonstration of a scaled down (100 W) P2P microgrid system to provide a low cost, modular, safe, portable testing environment for new EMS algorithms. The system nonetheless has realistic behaviour in terms of control interfaces, measurements, dynamics, and therefore provides a valuable insight into EMS implementation that cannot be obtained through simulations alone. In this work, three microgrid emulators were built and they can communicate with each other via TCP/IP, enabling development and demonstrations of distributed forecasting, control and optimization algorithms.
\end{abstract}

Index Terms-Battery storage systems, energy management systems, peer-to-peer, predictions, microgrid, decentralised, multi-agent, photovoltaic system.

\section{INTRODUCTION}

The use of fossil fuel-based energy has been identified as the largest source of overall anthropogenic greenhouse gases (GHG) emissions [1]. The COP21, also known as the 2015 Paris Climate Conference which involved 196 nations has formalised a legally binding and universal agreement on aiming to limit global warming to well below $2{ }^{\circ}$ Celsius (C) above preindustrial levels and to pursue efforts to limit the temperature increase to $1.5{ }^{\circ} \mathrm{C}$ [2]. In view of this, renewable energy technologies have been developed rapidly and they are being adopted from countries all over the world [3]. Globally, this is dominated by large (e.g., megawatt-scale and up) generators that are owned by utilities or large investors [4], although it has been shown that these are insufficient in providing electricity services to the energy-poor communities [5]. Meanwhile, the market for distributed small-scale generation, also known as microgrid has started to accelerate in both developing and developed countries. In developing countries, the rapid expansion of small-scale renewable systems is associated with

L. K. Gan, J. Lee, D. A. Howey are with the Department of Engineering Science, University of Oxford, Oxford, U.K. (e-mail: leong.gan@eng.ox.ac.uk, jaehwa.lee@eng.ox.ac.uk, and david.howey@eng.ox.ac.uk). B. Riar is with the Department of Electrical \& Computer Engineering, Utah State University, Utah, United States (e-mail: baljitriar@ieee.org). rural electrification for people living far from the grid. On the contrary, significant growth is observed in developed countries from residential and industry in response to a demand for selfsufficiency and the desire to become prosumers [4].

Because of stochastic behaviour of the renewable energy sources, relying solely on DGs is insufficient to maintain the power balance between the supply and demand [6]. These fluctuations may potentially cause stability problem and degradation on power quality, especially in a weak grid system [7]. The remedy to this problem is to use energy storage systems (ESS). Whenever more than one energy sources exist within a microgrid, an energy management system (EMS) is required to guide the power flow within a well-defined environment (physical system) efficaciously. Ideally, an EMS aims to maximise the utilisation of renewable energy, reduce the stress level experienced by ESS, minimise the cost of electricity and maintain the stability and reliability of the system by supplying the load in all conditions. In general, an EMS can be implemented either with conventional rule-based or intelligent-based strategies. The control architectures can be classified as central-hierarchical, distributed-hierarchical or complete decentralised (also known as peer-to-peer) [8]. The on-going research of distributed intelligent EMS for modern microgrids $[9]-[12]$ is enabling the realisation of smart grid systems [13], [14].

In principle, a bottom-up decentralised microgrid systems distinguish themselves from centralised power systems in terms of self-sufficiency, resiliency and scalability. In the former, no dedicated central or master control entity exists and each system has no complete information about the overall system state. Decisions are made based only on local information and hence, failure on one node will not ruin the entire system [15]. A fully decentralised structure is often coordinated by layers of software which are modularised horizontally, with each independent from one another to eliminate single points of failure [15]. The employment of Information and Communication Technology (ICT) is often required to enable interaction, collaboration and information sharing among distributed generators (DGs) through information exchange. In particular, the control entities need to communicate in a $\mathrm{P} 2 \mathrm{P}$ manner to accomplish these. The exchanged information is then processed by the local adaptable learning system and optimiser to achieve certain goals which are defined by users. Therefore, from the Smart Grid context, a control structure which is capable of communicating, learning and optimising can be simply described as an agent [8]. In a P2P microgrid system, a multi-agent system is distinctively defined as a control paradigm to carry out the EMS [8]. However, 
it is also worth noting that heavy reliance on Internet-like communication networks also exposes the system to cyberattack and vulnerability against the breach of privacy among users. To combat these, a distributed agent-based protection system which was proposed by the authors in literature [16] may be used.

Recently, a full-scale pure P2P dc microgrid was developed and tested in Okinawa, Japan [17]. Whilst it was not possible to test the communication system using simulation tools alone, the implemented real decentralised infrastructure involved considerably long debugging time and may not be ideal to be used as a platform to test new EMS algorithms. In the existing literature, EMS algorithms are usually verified with costly realtime digital simulators (RTDS) [18]-[20]. However, the verification of decentralised control algorithms is challenging in these simulators due to their limited portability, scalability and connectivity. Also, the simulated electrical behaviour may not be realistic, depending on the fidelity of the models used. In contrast, this work proposes the use of a real microgrid, scaled down in power. The designed test rig aims to provide a low cost, modular and portable testing environment for new EMS algorithms, which would be difficult to implement on larger systems. Realistic behaviour in terms of control, interfaces, measurements, dynamics can be monitored from the proposed system and hence, providing a valuable insight into the EMS implementation that cannot be obtained through simulations alone. To mitigate safety concerns, voltages and currents are kept low, and a simple fail-safe approach is incorporated to increase user confidence, with hardware (fuses, zener diodes) and low-level software (over-voltage, over-current) protection included.

This paper presents in the following structure. An overview of the proposed peer-to-peer microgrid emulator is described in Section II. The discussion on hardware, communication protocols, together with the protection mechanisms is given in Section III. Using the developed test-rig, the operating principles of the proposed microgrid emulator is demonstrated in Section IV. Finally, conclusions derived from the research are summarised in Section V.

\section{Distributed Microgrid System For EMS Testing}

The microgrid emulators were designed with the following key objectives:

1) Flexible in managing power flow between the components

2) Condition monitoring for safe operation

3) Information sharing between microgrids which allow coregional photovoltaic (PV) power and load predictions using probabilistic models

4) Allow EMS algorithms to be developed and tested in a realistic environment

To fulfil these objectives, a distributed microgrid infrastructure with the communication system is necessary. The overall block diagram of the microgrid emulator is shown in Fig. 1 . The photovoltaic panel and lead-acid battery are connected to the common DC bus via bespoke DC/DC converters. For simplicity, a power supply (EA-PS 3032-05 B) and an

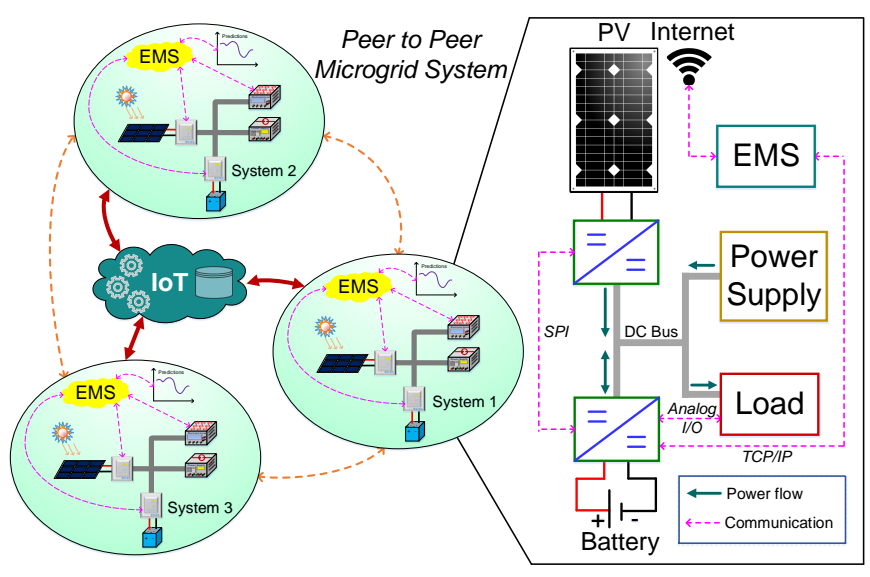

Fig. 1. Block diagram of the laboratory-scaled decentralised PV-battery system

electronic load (EA-EL 3160-60) are used to emulate the utility grid. The power supply is programmed to control the voltage of the common DC bus and it supplies the power deficit within the microgrid system. The electronic load is connected in parallel with the power supply to emulate the consumer load and grid export. If required, a realistic load profile can be emulated by varying the power consumption accordingly.

In this work, three microgrid emulators were built and they are able to communicate with each other via TCP/IP, enabling development and demonstrations of distributed forecasting, control and optimisation algorithms. An open cloud-based Internet of Things (IoT) platform, ThingSpeak is used for storing, retrieving and visualising data in real-time [21]. One of the main advantages of this platform is the integrated support of Matlab which allows data analysis programmes to be deployed in the cloud. Additional services by ThingSpeak include interaction with social media, web services, other devices and reacting to both raw data and new data. Measurements from each microgrid emulator can be stored and retrieved from a single channel through a dedicated application programming interface (API). This is especially useful and pragmatic for geographically dispersed systems, such as the distributed microgrid emulators proposed in this paper. Each emulator consists of a solar PV system, lead-acid storage system, power converters, temperature sensors, power supply, load and PC.

The employed software structure, which was designed on a modular basis is shown in Fig. 2. The physical layer (lowlevel programme) executes the control logics and instantiates communication link between the EMS algorithm and hardware. The low-level control logics mainly control the dcdc converters, provide current references for the electronic load, implement battery management systems, and protect the microgrid from safety limits. The second layer is equipped with Matlab software which provides a platform for EMS deployment. As part of the EMS, a relatively sophisticated battery model (for example the electrochemical model) may be deployed in the PC and users may perform online state-ofcharge (SOC), remaining capacity and internal resistance estimations [22]-[26]. The next level which is also known as the 


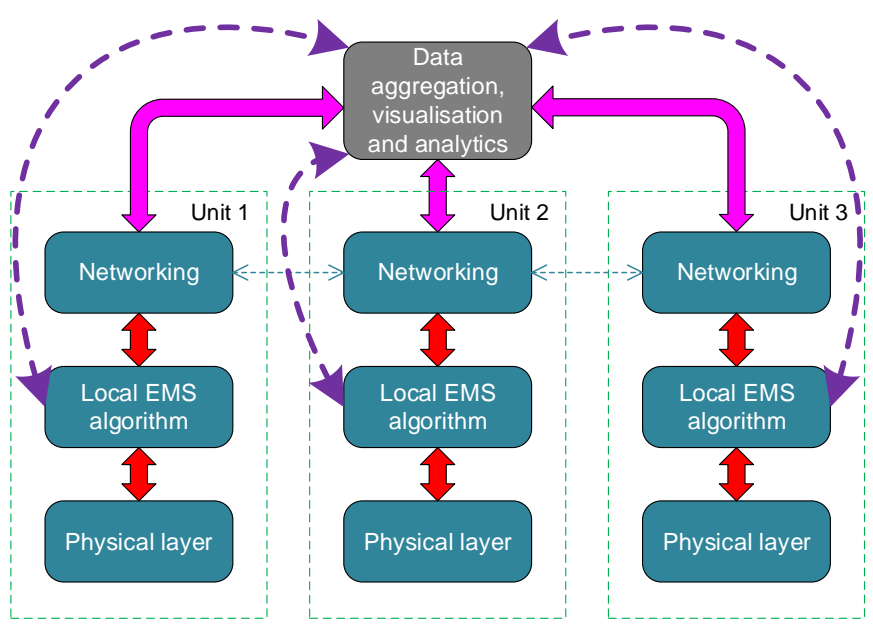

Fig. 2. Modular software structure

middleware, realises the direct P2P communications between the emulators. Several identified approaches are elaborated in literature [17]. Lastly, the upper-most layer is materialised with a cloud-based data analytics. In this paper, it is used for visualisation and it will be shown in the later section. A communication contingency is also considered whereby if the Networking layer is down, the second layer can directly communicate with the cloud without affecting the overall operation of the microgrids. Hence, the alternative communication paths enhance the resiliency of the microgrids.

The adopted microgrid configuration is the DC system, which has the advantages of not requiring synchronisation, frequency and phase control devices [27]. With the broad deployment of DC-based DGs such as solar PV panels and small wind turbines which are operating with permanent magnet generators that require rectification, the option of connecting consumer households to a DC bus system is viable [28]. In addition, the presence of plug-in electric and hybrid vehicle charging infrastructure increases the attractiveness of having the DC system [29]. However, an individual DC system cannot eliminate the losses in multiple stage conversions, though it is reported that the DC supplying system demonstrated has a greater efficiency than the standard AC one [30], [31].

\section{Modular Microgrid Design}

A hardware module which comprises of three pluggable boards for power conversion, control and communication are designed as demonstrated in Fig. 3 It is important to emphasise that the module is suitable to be used for any DC-based DGs, acting as a control and condition monitoring device and interact with other devices using through SPI, TCP/IP and Analogue I/O communication interfaces. The modular design approach accelerates and eases debugging process when a fault occurs as the boards can be isolated from each other. The following subsections further describe the design of the dc$\mathrm{dc}$ converter and its corresponding control topology, condition monitoring system, communication system and the protection scheme.

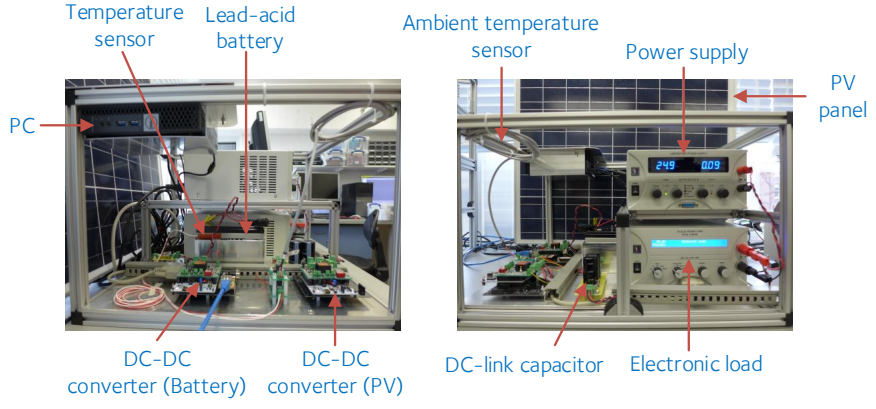

Fig. 3. Front and side view of the microgrid emulator

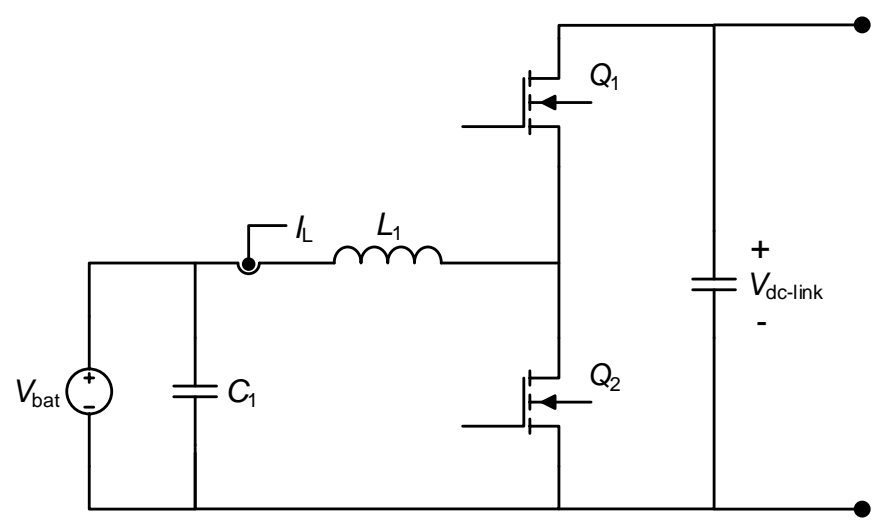

Fig. 4. Power stage of a bi-directional half-bridge dc-dc converter

\section{A. DC-DC converter and control scheme}

The bi-directional half-bridge dc-dc converter topology is used here to regulate the power in and out of the PV panel and battery. Both converters are identical for their power stages (shown in Fig. 4), each controlled by a dedicated ARM-based microcontroller (NUCLEO-F401RE) incorporating low-level PWM generation and PI-based inner current and outer voltage control schemes for the battery and PV panel. In order to extract the maximum power from the PV panel, the common maximum power pointing tracking (perturb and observe) algorithm [32] is also incorporated in the microcontroller. The converter circuit parameter is shown in Table 1. The output of the converters is connected to the DC link and the DC bus voltage which is set to $25 \mathrm{~V}$.

The corresponding PI-based controller block diagram is shown in Fig. 5. There are three main operating modes of the converter. The discharge mode, Mode 1 employs constantcurrent (CC) while the charging operation (Mode 2 or Mode 3) is governed by the constant current-constant voltage (CCCV) strategy [33]. In Mode 1, the control flow switch $S_{1}$ is in the upper position and the current reference, $I_{\text {ref_1 } 1}$ is used. This current reference is derived from the EMS power setpoint, $P_{\text {set-point }}$ and the battery voltage, $V_{\text {bat }}$. During charging operation, CC strategy is first utilised until an upper battery voltage limit is reached and the control flow switch, $S_{1}$ moves to lower position and activate $\mathrm{CV}$ charging strategy. The latter uses the reference current, $I_{\text {ref_2 }}$ which is generated from the voltage controller.

The BMS is further illustrated from the simplified flowchart of the main programme (combination of step 2 and 3 ) as shown 
TABLE I

SPECIFICATIONS OF THE DC-DC CONVERTER

\begin{tabular}{ll}
\hline \hline Parameters & Values \\
\hline Capacitance low voltage side & $30 \mu \mathrm{F}$ \\
Capacitance high voltage side & $9.4 \mu \mathrm{F}$ \\
Inductance & $82 \mu \mathrm{H}$ \\
Switching frequency & $80 \mathrm{kHz}$ \\
\hline \hline
\end{tabular}

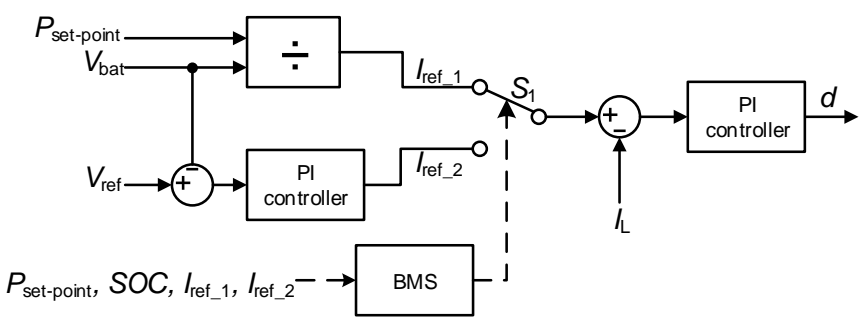

Fig. 5. Simplified control scheme of the dc-dc converter

in Fig. 6 It runs at a much higher sampling rate than the EMS and it is implemented as a state machine. The battery SOC is estimated with the coulomb counting method, which is governed by (1):

$$
\operatorname{SOC}[n]=\operatorname{SOC}[n-1]-\frac{I[n]}{Q} \Delta T
$$

Based on the current estimated SOC, the BMS decides whether the battery to operate either charging mode, discharging mode or stay idle. In addition, an appropriate power ramp rate is provided to obtain a smoother power transient. As it is acknowledged that a battery is recommended to operate within the predefined SOC limits, the software checks the current SOC value before deciding the appropriate state for the battery to operate (charging, discharging or idle).

The dc-dc converter power set-point is computed by the EMS, either via rule-based or real-time optimisation techniques. In the latter, one of the de-fecto industry standards is the model predictive control (MPC) algorithm. An MPC is a discrete-time control scheme which can be realised in a distributed manner [27]. Literature [34] proposed a distributed model predictive control approach to extract optimal power from the wind energy conversion systems, in a cooperative manner rather than competing with each other. Simulation results showed that the control scheme is especially useful in coordinating the load sharing between multiple DGs in a situation whereby ESS is limited or it runs out of its capacity, with the aim of stabilising grid DC voltage. Coupling with the intelligent-based prediction algorithms on DG generations and load consumption, the associated cost function can be optimised within a preceding horizon, subjected to various constraints. We do not cover the detail of those methods in this paper, but the decision of an appropriate set-point can be a key idea to enhance the economic performance of EMS.

\section{B. Condition monitoring and protection scheme for safe op- eration}

To safeguard the microgrid components from damage, a condition monitoring system is essential and it is required

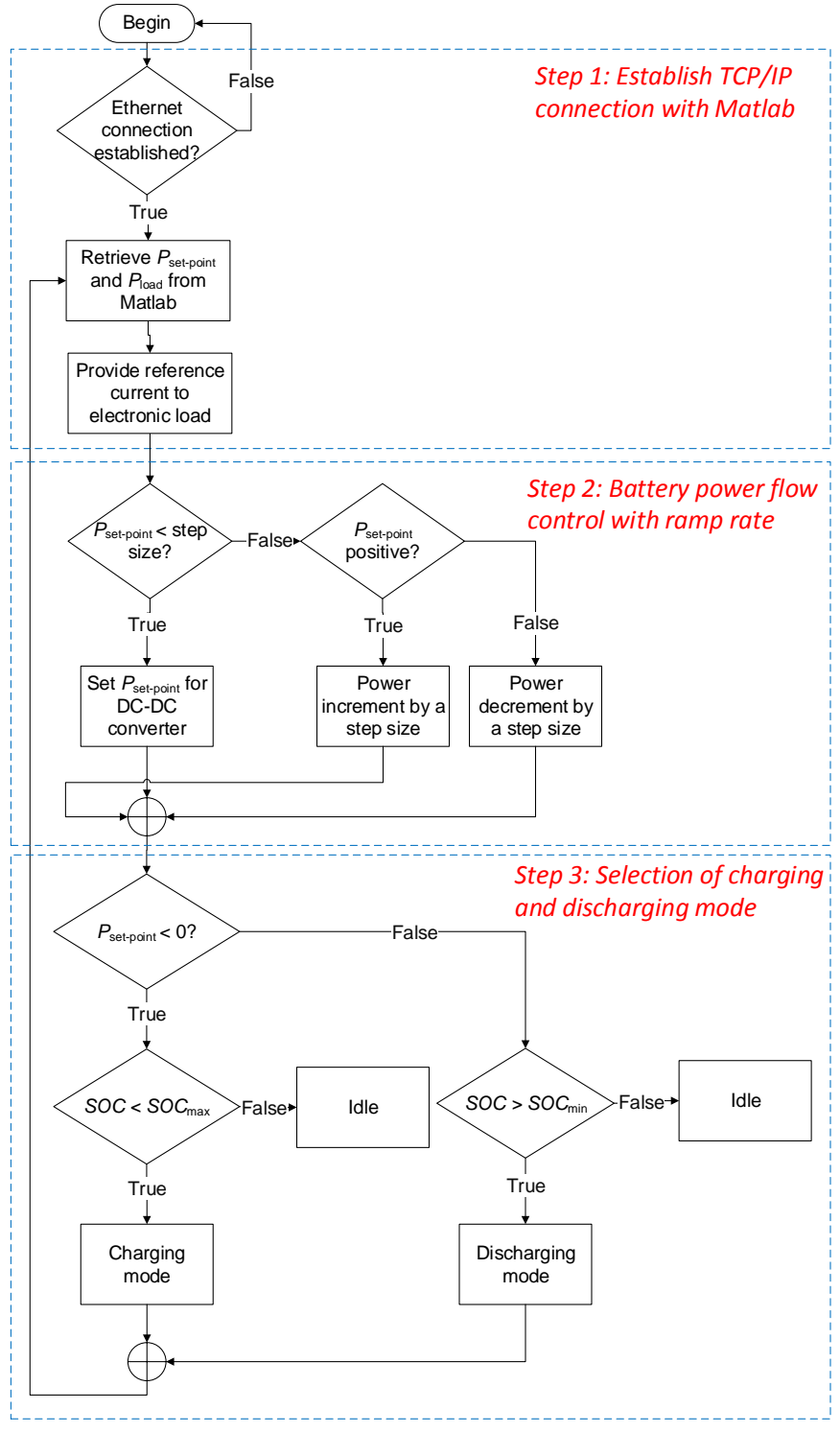

Fig. 6. Simplified flowchart of the microcontroller main programme

to observe the operating voltages, currents and temperature. These measurements are used as part of the protection scheme. In particular, the DC link voltage, battery voltage, battery current, PV voltage, PV current, load current as well as the ambient temperature and battery temperature are primarily measured. Coupling with the appropriate signal conditioning circuitries, these can be measured via analogue to digital converters (ADC), current sensors and resistance temperature detectors (RTDs). The block diagram of the condition monitoring system is shown in Fig. 7. Using the measured values, other parameters such as battery SOC, power flow, etc. may be derived and estimated accordingly.

A platinum resistor thermometer (Pt100) [35] is used to monitor the ambient temperature while the battery surface temperature is measured with the silicone rubber surface RTD [36]. The temperature range of the Pt100 is $0 \mathrm{C} 200 \mathrm{C}$ and the latter is $-50 \mathrm{C} 200 \mathrm{C}$. Both RTDs are configured as 3-wire systems. As the RTDs are used for remote temperature monitoring, they require current excitations, and this is pro- 


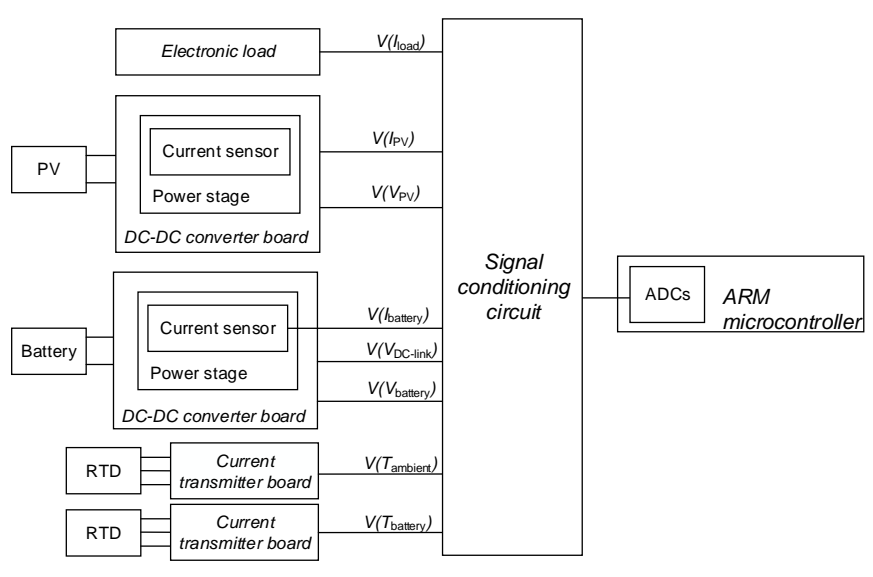

Fig. 7. Condition monitoring system

vided with the current transmitter by Texas Instruments [37].

The voltage and current protection schemes are formulated using both hardware and software. The over-current protection of the PV panel, battery and the power supply is performed by using the quick blow fuses. In addition, a zener diode is placed at the output of the dc-dc converter to prevent over-voltage. The protection sub-routine, as shown in Fig. 8 is executed immediately after obtaining the measurements from the ADCs. These include the inductor current, dc-link voltage, battery voltage and load power. The system will be immediately shutdown if any of the upper and lower safety limits are breached to avoid hazardous conditions. A unique error code is also assigned to each failure event to ease debugging process.

\section{Communication system}

Ultimately, it is desired to understand the flow of information throughout the microgrid as the success of an EMS relies on this. In particular, the rate of information exchange varies at different control hierarchies, and the corresponding timing effect on the ESS can be investigated with the customised communication scheme. If necessary, the timings can be optimised to suit a specific application. Besides, it is acknowledged that an efficient communication system is essential to the realisation of smart microgrid and the proposed prototype integrates several communication protocols to achieve this. These include a serial peripheral interface (SPI), TCP/IP and analogue interface. Fig. 9 shows the block diagram of the implemented communication interfaces. The SPI modules are featured within the ARM microcontroller while the TCP/IP communication is implemented with an embedded Ethernet module (W5500) and the analogue interface requires the support from ADC, digital-to-analogue converter (DAC) and digital I/O.

Information exchange between the dc-dc converters is carried out through the SPI interface. The battery converter acts as a master device and inquires the status of the PV converter (slave device) before enabling the MPPT algorithm. As part of the fail-safe operation, the PV converter is disabled whenever SPI communication link fails. The converter turns back on when the link is re-established. The electronic load is controlled via the 15-pole analogue interface, with the

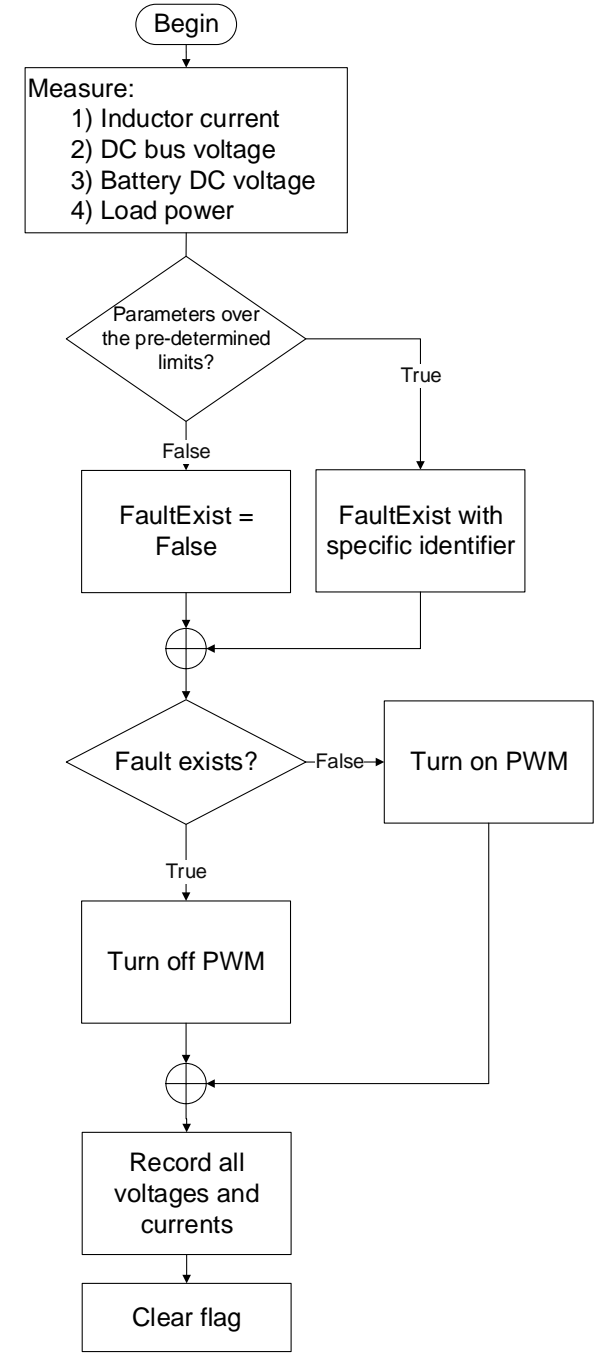

Fig. 8. Flowchart of the software protection scheme

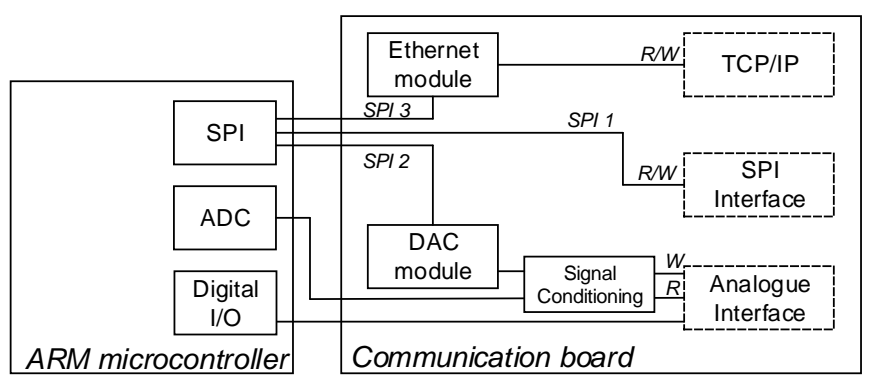

Fig. 9. Communication system

DAC performing write function while the ADC monitors the output of the electronic load. This straightforward and low cost communication interface allows load power value to be varied and at the same time the safety limit of the device is being ensured.

A compact local PC running Matlab allows the implementation of EMS algorithms that provide a power set-point to each microcontroller via the TCP/IP based Ethernet. The microcontrollers report back the operating conditions of the microgrid to the PC with parameters such as battery SOC, power flow, temperature, and voltages and currents of the DC/DC 

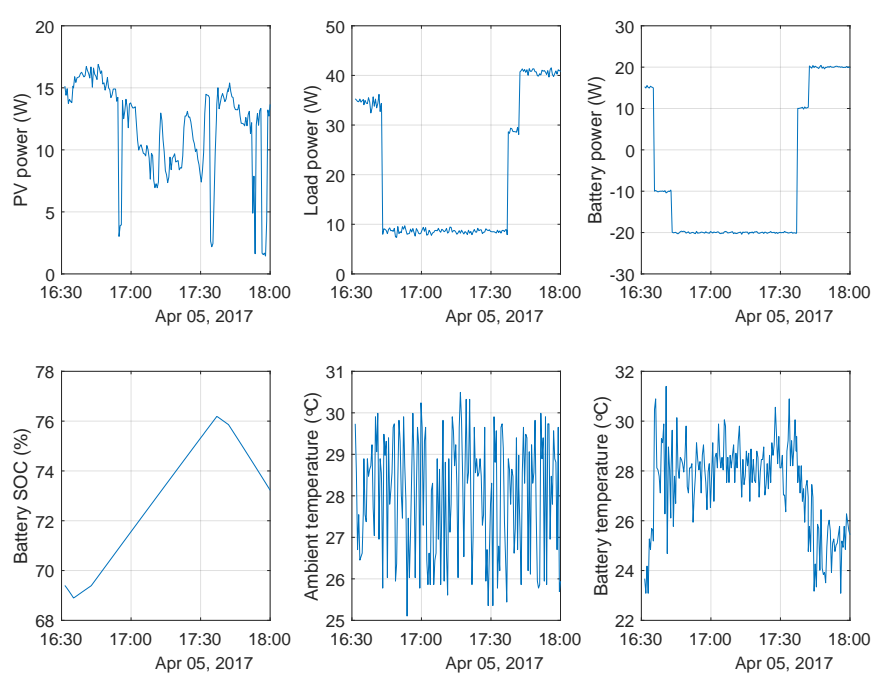

Fig. 10. Sample measurement results of $a$ ) PV power $b$ ) load power $c$ ) battery power $d$ ) battery SOC $e$ ) ambient temperature $f$ ) battery temperature with a sampling time of 30 seconds

converters. Similarly, the microgrid will not operate when the Ethernet communication is not established. It is acknowledged that the system network security may be compromised with the internet usage and the use of encryption algorithm is a potential area of improvement for such application.

\section{EXPERIMENTAL RESULTS}

An example of recorded data (every 30 seconds) for the microgrid system is given in Fig. 10 The PV output power fluctuates, with its peak reaching approximately $17 \mathrm{~W}$ (Fig. 10a). The load was initialised with a constant power consumption of $35 \mathrm{~W}$ (Fig. 10p) and the battery with an initial discharging of $15 \mathrm{~W}$ Fig. (10). At about 16:45, the load power was reduced to slightly less than $10 \mathrm{~W}$ and the battery was charging at $20 \mathrm{~W}$. The battery SOC (Fig. $10 \mathrm{~d}$ ) varied accordingly with its charging or discharging operation. The battery temperature started to increase during charging operation and decreased during discharging at around 17:30, as shown in Fig. 10] [38], [39].

In this work, the recorded data from second layer (Fig. 2) is transmitted directly to the ThingSpeak database. The microgrid emulators are assigned with different channels, with each consists of dedicated read and write API keys. Data from each channel can be assessed by other units as long as the correct read API key is used. The channels can be updated in real-time and they can be made for public viewing if desired. A sample screenshot of a channel for visualisation is shown in Fig. 11 As mentioned before, the channel data may be analysed in the cloud using Matlab toolboxes. When they meet certain conditions or with time control method, commands and alerts may be sent to devices and users, respectively. One potential application is to predict the microgrid state based on current measurements and notify operators before the limits are violated in order to prevent any blackouts.

\section{CONClusion}

This paper presents the development of three P2P microgrid emulators, enabling development and demonstrations

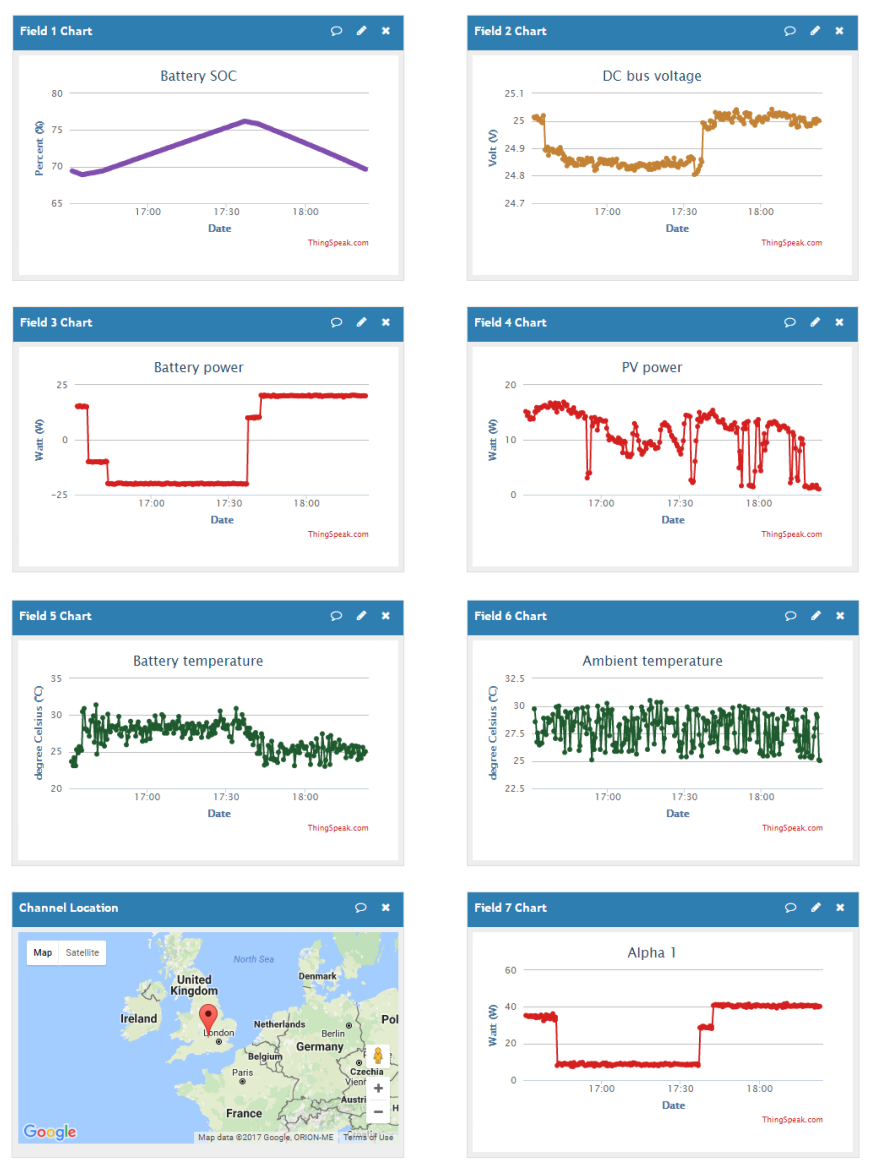

Fig. 11. A screenshot of a ThingSpeak channel

of distributed forecasting, control and optimisation for new EMS algorithms. As discussed, the software structure play an important role in realising a pure P2P microgrid system and researchers may use the proposed platform to test their EMS algorithms along with a realistic communication system. A condition monitoring was also incorporated to prevent the microgrid components from overloaded, whilst assisting users to debug their EMS software. Although the integrated IoT was not fully exploited in this paper, it is expected that IoT technologies will play a crucial role in the futuristic smart grid system. These include and not limited to data sharing, condition monitoring and encouraging social involvement in distributed power systems.

\section{ACKNOWLEDGEMENT}

This work is carried out as a part of the project Intelligent Management of Multiple Decentralised Solar/Energy Storage Systems, and is supported by the funding from Korea Institute of Energy Technology Evaluation and Planning (KETEP).

\section{REFERENCES}

[1] "CO2 Emissions from Fuel Combustion Highlights," International Energy Agency (IEA), 2015.

[2] "Energy, Climate Change and Environment: 2016 Insights," International Energy Agency (IEA), 2016.

[3] "World Energy Outlook 2015," International Energy Agency (IEA), 2015.

[4] "Renewables 2016 Global Status Report," REN21, 2016. 
[5] "Poor people's energy outlook 2016: National Energy Access Planning from the Bottom Up," Practical Action Publishing, 2016.

[6] J. Kaldellis, Stand-Alone and Hybrid Wind Energy Systems: Technology, Energy Storage and Applications. Woodhead Publishing, 2010.

[7] D. Weisser and R. S. Garcia, "Instantaneous wind energy penetration in isolated electricity grids: concepts and review," Renewable Energy, vol. 30, no. 8, pp. 1299-1308, 2005.

[8] G. Rohbogner, S. Fey, U. J. J. Hahnel, P. Benoit and B. WilleHaussmann, "What the term Agent stands for in the Smart Grid Definition of Agents and Multi-Agent Systems from an Engineer's Perspective," in Federated Conference on Computer Science and Information Systems, 2012, pp. 1301-1305.

[9] H. Aki, T. Wakui, and R. Yokoyama, "Optimal management of fuel cells in a residential area by Integrated-Distributed Energy Management System (IDEMS)," in 2016 IEEE Power \& Energy Society Innovative Smart Grid Technologies Conference (ISGT), pp. 1-5.

[10] X. Wu, X. Hu, X. Yin, and S. Moura, "Stochastic Optimal Energy Management of Smart Home with PEV Energy Storage," IEEE Transactions on Smart Grid, pp. 1-1, 2016.

[11] Y. Li and Y. Han, "A Module-Integrated Distributed Battery Energy Storage and Management System," IEEE Transactions on Power Electronics, pp. 1-1, 2016.

[12] R. Kurte, K. I.-K. Wang, D. Thrimawithana, U. K. Madawala, and Z. Salcic, "An intelligent hybrid communication system for a distributed renewable energy management," in IECON 2013 - 39th Annual Conference of the IEEE Industrial Electronics Society, pp. 3323-3328.

[13] P. Vrba, V. Marik, P. Siano, P. Leitao, G. Zhabelova, V. Vyatkin, and T. Strasser, "A Review of Agent and Service-Oriented Concepts Applied to Intelligent Energy Systems," IEEE Transactions on Industrial Informatics, vol. 10, no. 3, pp. 1890-1903, 2014.

[14] T. Strasser, F. Andren, J. Kathan, C. Cecati, C. Buccella, P. Siano, P. Leitao, G. Zhabelova, V. Vyatkin, P. Vrba, and V. Marik, "A Review of Architectures and Concepts for Intelligence in Future Electric Energy Systems," IEEE Transactions on Industrial Electronics, vol. 62, no. 4, pp. 2424-2438, 2015.

[15] A. S. Tanenbaum, Distributed Systems: Principles and paradigms, 2nd ed. Pearson Prentice Hall, 2007.

[16] K. J. Ross, K. M. Hopkinson, and M. Pachter, "Using a Distributed Agent-Based Communication Enabled Special Protection System to Enhance Smart Grid Security," IEEE Transactions on Smart Grid, vol. 4, no. 2, pp. 1216-1224, jun 2013.

[17] A. Werth, A. Andre, D. Kawamoto, T. Morita, S. Tajima, D. Yanagidaira M. Tokoro, and K. Tanaka, "Peer-to-peer Control System for DC Microgrids," IEEE Transactions on Smart Grid, pp. 1-1, 2016.

[18] J. Tian, Z. Liu, J. Shu, J. Liu, and J. Tang, "Base on the ultra-short term power prediction and feed-forward control of energy management for microgrid system applied in industrial park," IET Generation, Transmission \& Distribution, vol. 10, no. 9, pp. 2259-2266, 2016.

[19] T. Logenthiran, D. Srinivasan, A. M. Khambadkone, and H. N. Aung, "Multiagent System for Real-Time Operation of a Microgrid in RealTime Digital Simulator," IEEE Transactions on Smart Grid, vol. 3, no. 2, pp. 925-933, 2012.

[20] A. Merabet, K. Tawfique Ahmed, H. Ibrahim, R. Beguenane, and A. M. Y. M. Ghias, "Energy Management and Control System for Laboratory Scale Microgrid Based Wind-PV-Battery," IEEE Transactions on Sustainable Energy, vol. 8, no. 1, pp. 145-154, 2017.

[21] MathWorks, "ThingSpeak," 2017. [Online]. Available: https: //thingspeak.com/

[22] L. Zheng, L. Zhang, J. Zhu, G. Wang, and J. Jiang, "Co-estimation of state-of-charge, capacity and resistance for lithium-ion batteries based on a high-fidelity electrochemical model," Applied Energy, vol. 180, pp. 424-434, 2016.

[23] Z. Wei, S. Meng, K. J. Tseng, T. M. Lim, B. H. Soong, and M. SkyllasKazacos, "An adaptive model for vanadium redox flow battery and its application for online peak power estimation," Journal of Power Sources, vol. 344, pp. 195-207, 2017.

[24] Z. Wei, T. M. Lim, M. Skyllas-Kazacos, N. Wai, and K. J. Tseng, "Online state of charge and model parameter co-estimation based on a novel multi-timescale estimator for vanadium redox flow battery," Applied Energy, vol. 172, pp. 169-179, 2016.

[25] A. Tulsyan, Y. Tsai, R. B. Gopaluni, and R. D. Braatz, "State-of-charge estimation in lithium-ion batteries: A particle filter approach," Journal of Power Sources, vol. 331, pp. 208-223, 2016.

[26] Z. Deng, L. Yang, Y. Cai, H. Deng, and L. Sun, "Online available capacity prediction and state of charge estimation based on advanced data-driven algorithms for lithium iron phosphate battery," Energy, vol. 112, pp. 469-480, 2016.
[27] A. Chauhan and R. Saini, "A review on Integrated Renewable Energy System based power generation for stand-alone applications: Configurations, storage options, sizing methodologies and control," Renewable and Sustainable Energy Reviews, vol. 38, pp. 99-120, 2014.

[28] K. Yukita, "Chapter 10 AC/DC microgrids," in Integration of Distributed Energy Resources in Power Systems, 2016, pp. 236-260.

[29] M. Yilmaz and P. T. Krein, "Review of Battery Charger Topologies, Charging Power Levels, and Infrastructure for Plug-In Electric and Hybrid Vehicles," IEEE Transactions on Power Electronics, vol. 28 , no. 5, pp. 2151-2169, may 2013

[30] G. A. L. Mitcheson and P., "Comparison of cost and efficiency of DC versus AC in office buildings," Report for Transformation of the Top and Tail of Energy Networks, Imperial College London, 2014.

[31] H. Kakigano, M. Nomura, and T. Ise, "Loss evaluation of DC distribution for residential houses compared with AC system," in The 2010 International Power Electronics Conference (ECCE ASIA), pp. 480-486.

[32] E. Koutroulis, K. Kalaitzakis, and N. Voulgaris, "Development of a microcontroller-based, photovoltaic maximum power point tracking control system," IEEE Transactions on Power Electronics, vol. 16, no. 1, pp. 46-54, 2001.

[33] D. Linden and T. B. Reddy, Eds., Linden's Handbook of Batteries, 4th ed. McGraw-Hill.

[34] P. Kou, L. Deliang, and L. Gao, "Distributed Coordination of Multiple PMSGs in an Islanded DC Microgrid for Load Sharing," IEEE Transactions on Energy Conversion, pp. 1-1, 2017.

[35] "Datasheet - Temperature probes Thermocouple / Pt100," 2015

[36] "Datasheet - Silicone Rubber Surface RTD Sensor," 2015.

[37] "Datasheet - 4-20mA Current Transmitter with Sensor Excitation and Linearization (XTR105)," 2004.

[38] M. V. Goran Kujundzic, Šandor Ileš, Jadranko Matuško, "Optimal charging of valve-regulated lead-acid batteries based on model predictive control," Applied Energy, vol. 187, pp. 189-202, may 2017.

[39] D. Rand and P. Moseley, "3 Leadacid battery fundamentals," in LeadAcid Batteries for Future Automobiles, 2017, pp. 97-132. 